58 Schacher, S., Castellucci, V.F. and Kandel, E.R. (1988) Science 240, 1667-1669

59 Dash, P.K., Moore, A.N. and Dixon, C.E. (1995) J. Neurosci. 15, 2030-2039

60 Schulman, H. (1995) Curr. Opin. Neurobiol. 5, 375-381

61 Moore, A.N., Waxham, M.N. and Dash, P.K. (1996) J. Biol. Chem. 271, 14214-14220

62 Blendy, J.A. et al. (1995) Brain Res. 681, 8-14

63 Deisseroth, K., Bito, H. and Tsien, R.W. (1996) Neuron 16, 89-101

64 Das, S. et al. (1997) Synapse 25, 227-233
65 Seidenbecher, T., Reymann, K.G. and Balschun, D. (1997) Proc. Natl. Acad. Sci. U. S. A. 94, 1494-1499

66 Gustafsson, B. and Wigström, H. (1990) Prog. Brain Res. 83, 223-232

67 Morris, R.G.M. and Frey, U. (1997) Philos. Trans. R. Soc. London 352, 1489-1503

68 Nadel, L. and Moscovitch, M. (1997) Curr. Opin. Neurobiol. 7 , 217-227

69 Stäubli, U. and Chun, D. (1996) J. Neurosci. 16, 853-860

70 Clark, K.A. and Collingridge, G.L. (1996) J. Physiol. 490, $455-462$

\title{
Language within our grasp
}

\author{
Giacomo Rizzolatti and Michael A. Arbib
}

In monkeys, the rostral part of ventral premotor cortex (area F5) contains neurons that discharge, both when the monkey grasps or manipulates objects and when it observes the experimenter making similar actions. These neurons (mirror neurons) appear to represent a system that matches observed events to similar, internally generated actions, and in this way forms a link between the observer and the actor. Transcranial magnetic stimulation and positron emission tomography (PET) experiments suggest that a mirror system for gesture recognition also exists in humans and includes Broca's area. We propose here that such an observation/execution matching system provides a necessary bridge from 'doing' to 'communicating', as the link between actor and observer becomes a link between the sender and the receiver of each message.

Trends Neurosci. (1998) 21, 188-194

'In all communication, sender and receiver must be bound by a common understanding about what counts; what counts for the sender must count for the receiver, else communication does not occur. Moreover the processes of production and perception must somehow be linked; their representation must, at some point, be the same.'

WHAT IS SAID HERE by Alvin Liberman ${ }^{1}$ for $\checkmark$ speech where individuals have an explicit intent to communicate, must apply also for 'communications' in which such an overt intention is absent. We understand when one individual is attacking another or when someone is peacefully eating an apple. How do we do it? What is shared by the (involuntary) sender and by the receiver? Is this mechanism the precursor of willed communications? The present review addresses these questions.

Giacomo Rizzolatti

is at the Istituto di

Fisiologia Umana

Università di

Parma, Via

Gramsci 14,

43100 Parma

Italy, and

Michael A. Arbib

is at the USC

Brain Project,

University of

Southern

California,

Los Angeles,

CA 90089-2520

USA

\section{The mirror system}

Neurons located in the rostral part of monkey inferior area 6 (area F5) discharge during active movements of the hand or mouth, or both ${ }^{2-4}$. Some years ago we found that in most F5 neurons, the discharge correlates with an action, rather than with the individual movements that form it ${ }^{3}$. Accordingly, we classified F5 neurons into various categories corresponding to the action associated with their discharge. The most common are: 'grasping with the hand' neurons, 'holding' neurons and 'tearing' neurons ${ }^{3,5}$. Further study revealed something unexpected: a class of F5 neurons that discharge not only when the monkey grasped or manipulated the objects, but also when the monkey observed the experimenter making a similar gesture $^{6-8}$. We called the neurons endowed with this property 'mirror neurons' (Fig. 1).

The response properties of mirror neurons to visual stimuli can be summarized as follows: mirror neurons do not discharge in response to object presentation; in order to be triggered they require a specific observed action. The majority of them respond selectively when the monkey observes one type of action (such as grasping). Some are highly specific, coding not only the action aim, but also how that action is executed. They fire, for example, during observation of grasping movements, but only when the object is grasped with the index finger and the thumb.

All mirror neurons show visual generalization: they discharge when the agent of the observed action (typically a hand) is far away from or close to the monkey. A few neurons respond even when the object is grasped by the mouth. The actions most represented are: grasp, manipulate, tear, and put an object on a plate. Mirror neurons also have motor properties that are indistinguishable from those of F5 neurons that do not respond to action observation. In this review, they will be referred to collectively and regardless of their other properties, as 'canonical neurons'. Typically, mirror neurons show congruence between the observed and executed action. This congruence can be extremely strict, that is, the effective motor action (for example, precision grip) corresponds with the action that, when seen, triggers the neuron (that is, precision grip). For other neurons the congruence is broader: the motor requirements (for example, precision grip) are usually stricter than the visual ones (for example, any type of hand grasping). An example of a highly congruent mirror neuron is shown in Fig. 2. What is 
Fig. 1. An example of a mirror neuron. The behavioral situation is schematically represented in the upper part of each panel. The responses of the neuron are shown in the middle and lower parts of each panel. The responses are shown as discharges using ten individual behavioral trials (each short vertical line corresponds to an action potential) and expressed as relative-response histograms. (A) The experimenter grasps a piece of food with his hand then moves it toward the monkey, who, at the end of the trial, grasps it. The neuron discharges during observation of the grip, ceases to fire when the food is given to the monkey and discharges again when the monkey grasps it. (B) The experimenter grasps the food with a tool. The subsequent sequence of events is as in (A). Note the lack of response of the neuron when the food is grasped with the tool. (C) The monkey grasps food in darkness. In (A) and (B) the rasters are aligned with the moment when the food is grasped by the experimenter (vertical line). In (C) the alignment is with the approximate beginning of the grasping movement. Each small vertical line in the rasters corresponds to a spike. Histogram bin width: 20 ms. Reproduced with permission from Ref. 7.

the function of mirror neurons? The proposal that we $^{7,8}$ and others ${ }^{9}$ have advanced is that their activity 'represents' actions. This representation can be used for imitating actions and for understanding them. By 'understanding' we mean the capacity that individuals have to recognize that another individual is performing an action, to differentiate the observed action from other actions, and to use this information to act appropriately. According to this view, mirror neurons represent the link between sender and receiver that Liberman postulated in his motor theory of speech perception as the necessary prerequisite for any type of communication ${ }^{1,10,11}$.

\section{What is area F5?}

Although doubts have been expressed ${ }^{12}$, most authors share the view that the rostral part of the monkey ventral premotor cortex (area F5) is the monkey homolog of Broca's area in the human brain. The reasons for this view are: that both F5 and Broca's area are parts of inferior area 6 (Refs 13-15) and their location within the agranular frontal cortex is similar (Box 1); and cytoarchitectonically, there are strong similarities between area 44 (the caudal part of Broca's area) and F5 (Refs 14,16,17).

Functionally, a difference between Broca's area and F5 is that Broca's area is most commonly thought of as an area for speech, whereas F5 is often considered as an area for hand movements. F5 is somatotopically organized - its dorsal part contains a representation of hand movements ${ }^{2,3,18}$ and its large ventral part contains a representation of mouth and larynx movement ${ }^{19,20}$; a similar organization is present in the ventral premotor cortex of other primates ${ }^{21}$. Similarly, the motor properties of human Broca's area do not relate only to speech: recent PET data indicate that Broca's area might also become active during the execution of hand or arm movements ${ }^{22,23}$, during mental imagery of hand grasping movement (mostly area 44$)^{24,25}$, and during tasks involving hand-mental rotations (areas 44 and 45$)^{26}$. Finally, Broca's area becomes active in patients who have recovered from subcortical infarctions when they are asked to use their paralyzed hand ${ }^{27}$.

It is intriguing that the area, which in the monkey contains a system that links action recognition and action production, is precisely that area that, for completely different reasons, has been proposed as the homologue of Broca's area. Is this a mere coincidence?
A
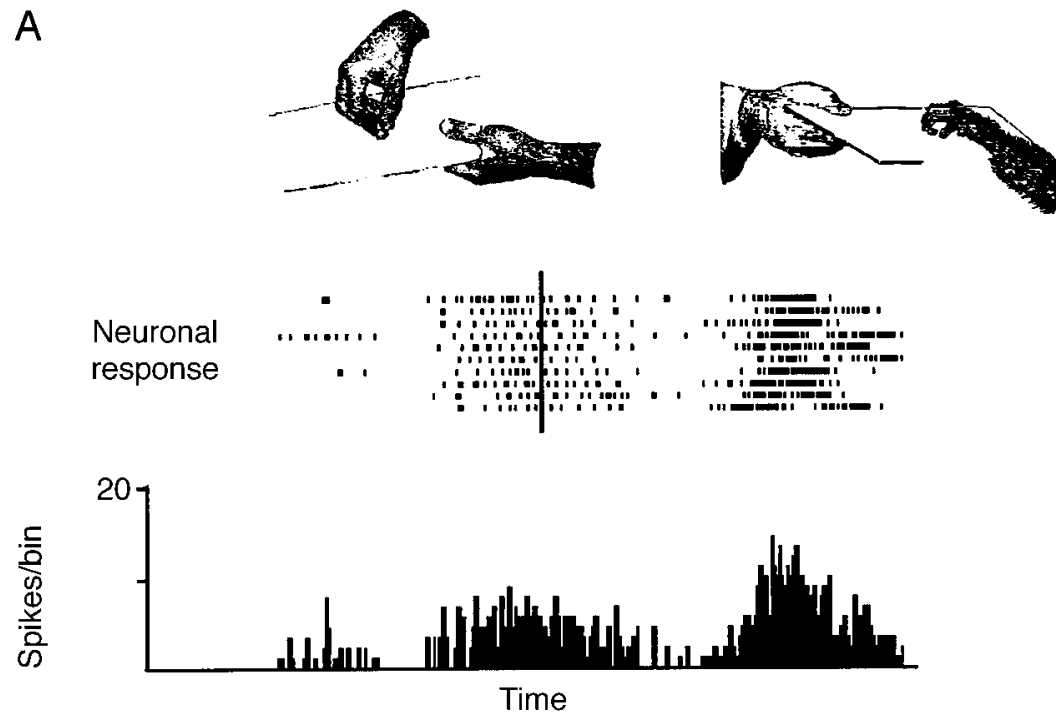

B
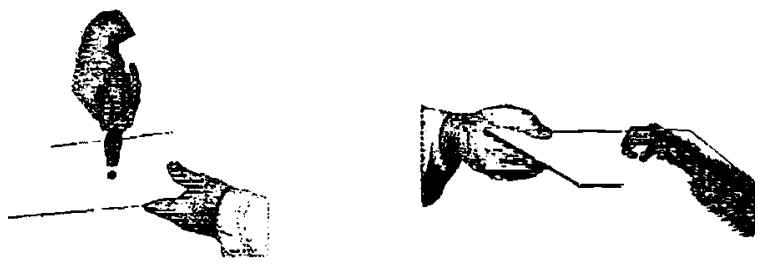

Neuronal

response
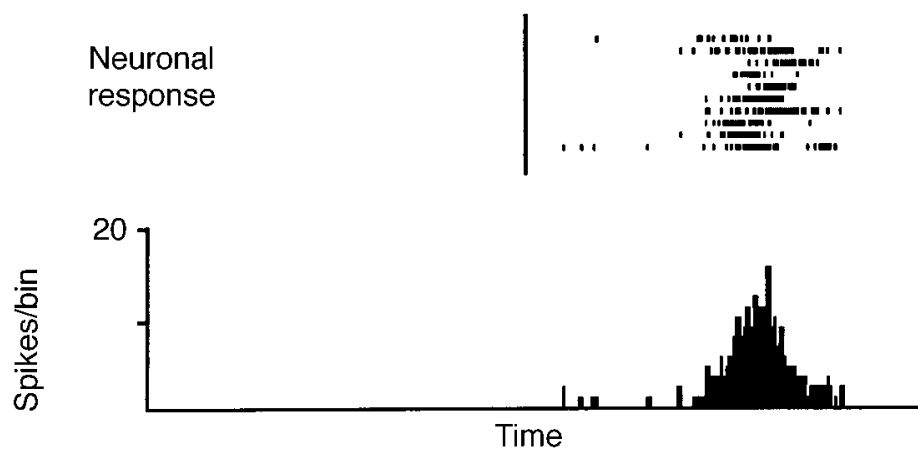

C

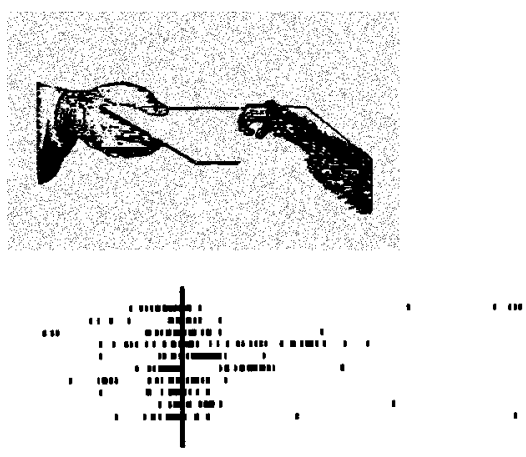

Neuronal

response

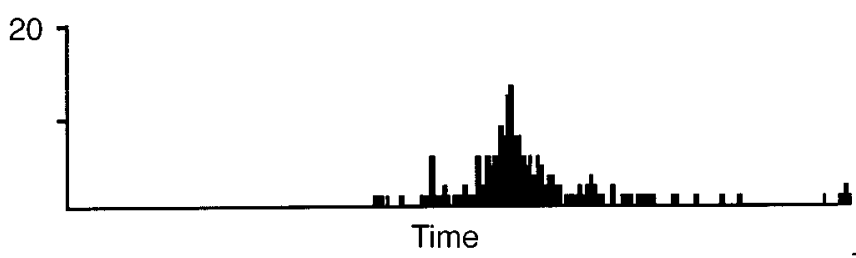

Or, on the contrary, has the mirror system been fundamental for the development of speech and, before speech, of other forms of intentional communication? Before discussing these points, we examine the evidence for a mirror system in humans. 
A

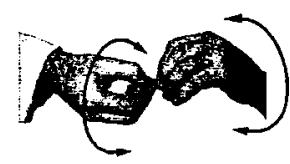

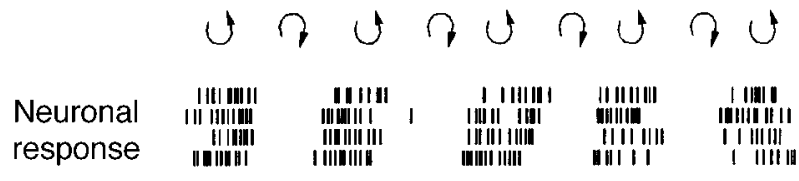

B
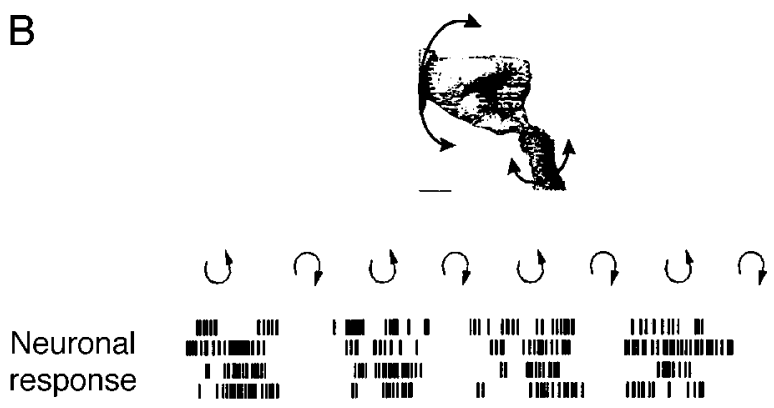

C

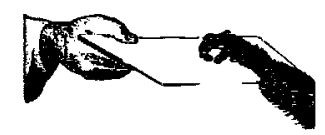

Neuronal

1

$\begin{array}{ll}1 \\ 1 & 1\end{array}$

$\begin{array}{lll}11 & 1 \\ 11 & 1 & 1\end{array}$

1

$1 \mathrm{~s}$

Fig. 2. An example of a highly congruent mirror neuron. The behavioural situation is schematically represented in the upper part of each panel. The responses of the neuron are shown in the middle and lower parts of each panel; four sketches of continuous recordings are shown in each panel, with each vertical line corresponding to an action potential. (A) The monkey observes the experimenter who rotates his hand around an object in opposite directions as to break it. The neuronal response is present in one rotation direction only. (B) The monkey rotates a piece of food held by the experimenter who opposes the monkey movement making a rotation in the opposite direction. (C) The monkey grasps food using the same finger as during rotation. Small arrows above the records (A and $\mathrm{B}$ ) indicate the directions of rotations. Reproduced with permission from Ref. 7.

\section{The mirror system in humans}

The first demonstration of a mirror system in humans was provided by Fadiga et al. ${ }^{28}$ The rationale of their experiment was the following: if the observation of an action activates the premotor cortex in humans as it does in monkeys, then magnetic transcranial stimulation should induce, during action observation, an enhancement of motor-evoked potentials recorded from those muscles that are active when the observed action is executed. Their results confirmed the hypothesis: during the observation of various actions, a selective increase of motor evoked potentials occurred in the muscles that the subjects usually use for producing them.

Although these data indicate that an action production and action observation matching system exists in humans, they do not give information on the circuits that underlie it. Data on this issue were provided by two PET experiments ${ }^{25,29}$. The two experiments differed in many aspects, but both had a condition in which subjects observed the experimenter grasping a 3-dimensional object; object observation was used as a control situation. Grasp observation significantly activated the superior temporal sulcus (STS), the inferior parietal lobule and the inferior frontal gyrus (area 45); all activations were in the left hemisphere.

Hemispheric differences aside, the cortical areas active during action observation in humans match well with those active in the monkey under the same conditions. Neurons that become selectively active in the STS during the sight of hand actions were described by Perrett and his co-workers ${ }^{30,31}$. There is evidence, although limited, that mirror neurons might be present in area 7b in monkeys (Ref. 32). Finally, as discussed earlier, it is likely that F5 is the monkey homolog of Broca's area.

Taken together, human and monkey data indicate that, in primates, there is a fundamental mechanism for action recognition. We argue that individuals recognize actions made by others because the neural pattern elicited in their premotor areas during action observation is similar to that internally generated to produce that action. This mechanism in humans is circumscribed to the left hemisphere. In the next section we will posit that this action-recognition mechanism has been the basis for language development.

\section{Action recognition and communication}

Animals' calls and human speech are undoubtedly different phenomena. Among the many aspects that differentiate them is a marked difference in the anatomical structures underlying the two behaviors. Animals calls are mediated primarily by the cingulate cortex plus some diencephalic and brain stem structures $^{33,34}$. Speech is mediated essentially by a circuit whose main nodes are the classical Broca's and Wernicke's areas, both located on the lateral cortical surface.

Our proposal is that the development of the human lateral speech circuit is a consequence of the fact that the precursor of Broca's area was endowed, before speech appearance, with a mechanism for recognizing actions made by others. This mechanism was the neural prerequisite for the development of interindividual communication and finally of speech. We thus view language in a more general setting than one that sees speech as its complete basis.

There is obviously an enormous gap between recognizing actions and sending messages with communicative intent. We offer now a hypothesis (for an earlier version, see Ref. 35) on how this gap might have been bridged. Whether an individual is about to perform an action or observes another individual performing an action, premotor areas are activated. Normally, a series of mechanisms prevents the observer from emitting a motor behavior that mimics the observed one, and the 'actor' from initiating the action prematurely. In the case of action observation, for example, there is a strong spinal cord inhibition that selectively blocks the motoneurons involved in the observed action execution (L. Fadiga, pers. commun.). Sometimes, however, for example when the observed action is of particular interest, the premotor 


\section{Box I. Cytoarchitectonic map of the caudal part of the monkey frontal lobe and possible homologies with human frontal cortex}

Figure A shows parcellation of prearcuate cortex ${ }^{\mathrm{a}}$ and agranular frontal cortex ${ }^{\mathrm{b}}$ of the macaque monkey and Fig. $\mathrm{B}$ shows parcellation of the region of the human frontal cortex defined as 'intermediate precentral cortex' by Campbellc. The terminology of Foerster ${ }^{\mathrm{d}}$ and Vogt and $\operatorname{Vogt}^{\mathrm{e}}$ has been adopted for the human cortex. Similar colors in A and B indicate areas with anatomical and functional homologies. Brain regions colored yellow are areas with anatomical and functional homologies, mostly related to orienting behavior; areas colored red also share anatomical and functional homologies and are mostly related to interactions with the external world ${ }^{\mathrm{e}-\mathrm{h}}$.
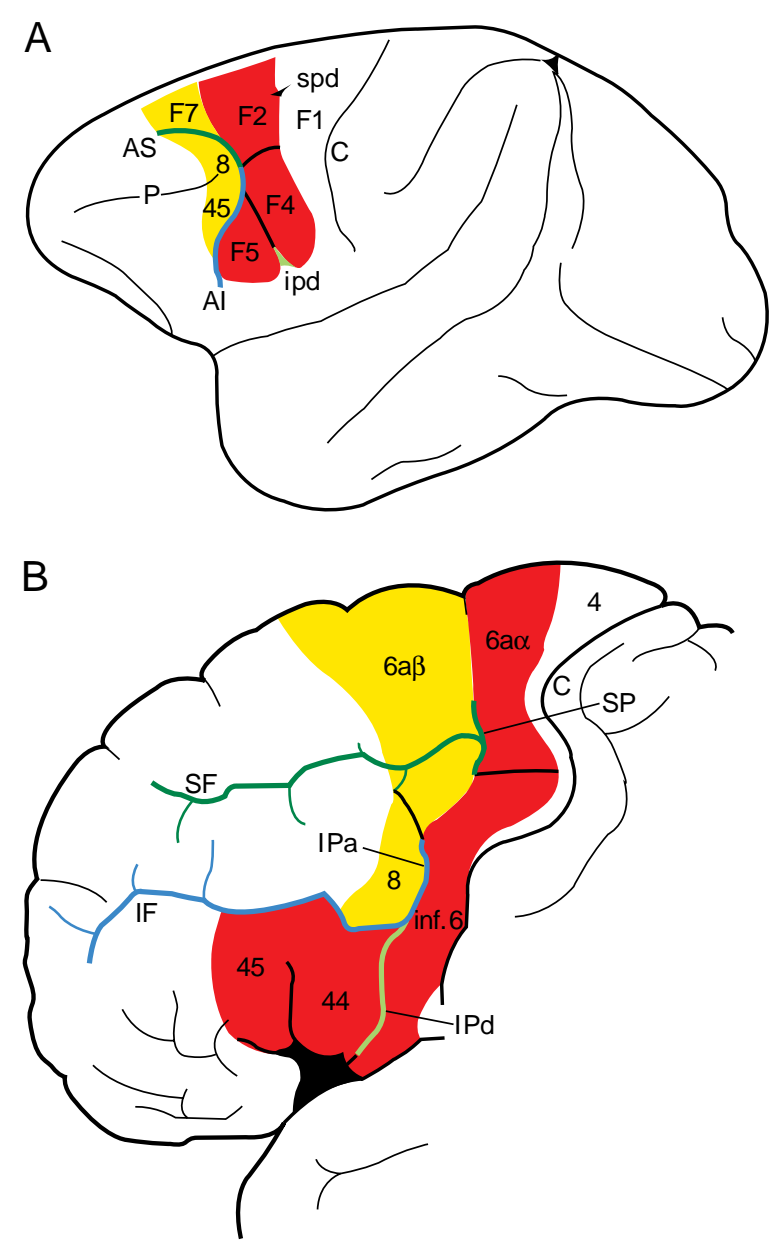

The homology is based on cytoarchitectonics, electrical stimulation $^{\mathrm{i}}$ and sulci embryology ${ }^{\mathrm{j}}$.

The superior frontal sulcus (SF) and the superior precentral sulcus (SP) of human brain are drawn in dark green as the superior limb of the monkey arcuate sulcus (AS). The inferior frontal sulcus (IF) and the ascending branch of the inferior precentral sulcus (IPa) of human brain are drawn in blue as the inferior limb of the monkey arcuate sulcus (AI). The descending branch of the inferior precentral sulcus (IPd) of human brain is drawn in pale green and is labeled as the inferior precentral dimple (ipd) of the monkey brain Fig. A. The reasons for these homologies are the following. The precentral sulcus develops from two separate primordia. Both of them have, during development, a horizontal branch representing the primordia of SF and IF, respectively. Typically, in the adult brain, the precentral sulcus ${ }^{i}$ is formed by two separate segments. Thus, we suggest that the human homolog of the monkey arcuate sulcus is formed by SF plus SP (dark green) and by the IF plus IPa (blue). The descending branch of inferior precentral sulcus (IPd, pale green) corresponds, in this view, to the inferior precentral dimple of the monkey. In humans it abuts IF. The proposed sulcal equivalence fits well the available data on the anatomical and functional organization of the premotor cortices in the two species. The equivalence between human IPd and monkey ipd is well supported by the fact that this sulcus marks the border between $\mathrm{F} 4$ and F5 in monkey and the border between inferior area 6 (inf. 6) and area 44 in humans. Abbreviations: 4, cortical area 4; C, central sulcus; F1, cortical area F1; P, principal sulcus; spd, superior precentral dimple.

\section{References}

a Walker, A.E. (1940) J. Comp. Neurol. 262, 256-270

b Matelli, M. , Luppino, G. and Rizzolatti, G. (1985) Behav. Brain Res. 18, 125-137

c Campbell, A.W. (1905) Histological Studies on the Localisation of Cerebral Function, Cambridge University Press

d Foerster, O. (1936) Brain 59, 135-159

e Vogt, C. and Vogt, O. (1926) Naturwissenschaften 14 1190-1194

f Bruce, C.J. (1988) in Neurobiology of Neocortex (Rakic, P. and Singer, W., eds), pp. 297-329, Wiley

g Suzuki, H. and Azuma, M. (1983) Exp. Brain Res. 53, $47-58$

h Matelli, M. and Luppino, G. (1992) Exp. Brain Res. (Suppl.) 22, 85-102

i Preuss, T.M., Stepniewska, I. and Kaas, J.H. (1996) J. Comp. Neurol. 371, 649-676

j Ono, M., Kubik, S. and Abernathey, C.D. (1990) Atlas of the Cerebral Sulci, Thieme system will allow a brief prefix of the movement to be exhibited. This prefix will be recognized by the other individual. This fact will affect both the actor and the observer. The actor will recognize an intention in the observer, and the observer will notice that its involuntary response affects the behavior of the actor. The development of the capacity of the observer to control his or her mirror system is crucial in order to emit (voluntarily) a signal. When this occurs, a primitive dialogue between observer and actor is established. This dialogue forms the core of language. The capacity to notice that one has emitted a signal and associating it with changes of the behavior of others might or might not have developed simultaneously. However, there is no doubt that, once established, this new association should have yielded enormous benefits of adaptive value for the group of individuals that started to make use of it, providing the selective pressure for the extension of communicative capacities to larger groups.

This new use of the mirror system, at both individual and species levels, marks the beginning of intentional communication. What actions were used for this new function in primates? Hand gestures or oro-facial movements? Before examining this issue, it is necessary to examine whether or not a 'prelinguistic grammar' can be assigned to the control and observation of actions. If this is so, the notion that evolution could yield a language system 'atop' of the action system becomes much more plausible. 


\section{A pre-linguistic 'grammar' of action in the monkey brain}

In order to provide abstract expression of the 'meanings' of neural activity in premotor cortex (monkey area F5), we have chosen 'case grammar' as a representation of sentence structure. Case grammar organizes sentences around action frames with slots for different roles. The key paper for case structure is 'The Case for Case' by Fillmore ${ }^{36}$, although many of the ideas about case have now been absorbed in the thematic structure of the lexicon that is an integral component of the Chomskian approach to syntax known as 'government and binding theory ${ }^{\prime 37}$. In a case analysis, the sentence 'John hit Mary with his hand' is viewed as the 'surface structure' for a case structure 'hit' (John, Mary, John's hand), which is an instance of the case frame 'hit' (agent, recipient, instrument), which makes explicit the roles of 'John', 'Mary' and 'John's hand'. Clearly, many different sentences can express the underlying case structure. Our analysis will deal with the two main types of F5 neurons: the mirror neurons and the 'canonical' F5 neurons.

(1) Imperative structure for 'canonical' F5 neurons. We view the activity of 'canonical' F5 neurons as part of the code for an imperative case structure, for example,

\section{Command: grasp-A(raisin)}

as an instance of grasp-A(object), where grasp-A is a specific kind of grasp, to be applied to the raisin. Note that this case structure is an 'action description', not a linguistic representation. 'raisin' denotes the specific object towards which the grasp is directed, whereas grasp-A is a specific command directed towards an object with well specified physical properties, but whose semantic properties are unspecified. The idea is that grasp type generalizes across similar grasps of varied objects has been postulated in opposition space theory ${ }^{38}$. Note that the slots in a case frame come with restrictions on what can fill those slots, for example, any $\mathrm{x}$ in grasp-A (x) must be a small object. As actions become more refined and as the transition to language occurs, the constraints on slot fillers might become more rigorous. From this, it follows that if the same principle holds for linguistic commands as for motor commands, Broca's area would code 'verb phrases' and constraints on the noun phrases that can fill the slots, but not details of the noun phrases themselves. This knowledge (objects or noun phrases) could be completely outside F5 or Broca's area; for example, in the temporal lobe.

(2) Declarative structure for mirror neurons. Having viewed the activity of canonical F5 neurons as coding a command (compared with an imperative sentence), we might say that the firing of 'mirror' F5 neurons is part of the code for a declarative case structure, for example,

\section{Declaration: grasp-A(Luigi, raisin)}

which is a special case of grasp-A(agent, object), where grasp-A is a specific kind of grasp, applied to the raisin (the object) by Luigi (the agent). Again, this is an 'action description', not a linguistic representation. If attention is focused on the agent's hand, then the appropriate case structure would be grasp-A (hand, object) as a special case of grasp-A (instrument, object). Thus, the same act can be perceived in different ways: 'who' grasps versus 'what' grasps. An interesting aspect of mirror-neuron properties is that they do not fire when the monkey observes the experimenter grasping the raisin with pliers, rather than his hand (Fig. 1B). However, after repetitive observation, a response to the tool may appear (G. Rizzolatti, unpublished). We thus see the ability to learn new constraints on a case slot: in this case the observed generalization of the 'instrument' role from hands alone to include pliers.

(3) Declarative structure for non-mirror 'canonical' F5 neurons. In the case of grasp-A (object), once the grasp is initiated, can it be asserted that the activity in F5 now becomes part of the declarative 'grasp-A (self, object)'? This is not an easy question. The neuronal discharge observed after hand-shaping onset might have command functions. It might, for example, reinforce the initial command to open the hand, or command the hand closure. Some 'canonical' F5 neurons, however, when tested passively, show proprioceptive responses $^{3,4}$. For these neurons, the discharge that accompanies the hand movement might have a specific declarative function concerning the agent of the action. The sentence in this case would be 'grasp-A (self, object)'.

\section{From action to speech}

Returning to our previous query as to which was the initial communicative gestural system in primates, we now distinguish between systems that are closed in the sense that they have a small, fixed repertoire and systems whose elements can be combined to yield an open repertoire of meaning. We argue that: (1) the mimetic capacity inherent to F5 and Broca's area had the potential to produce various types of closed systems related to the different types of motor fields present in that area (hand, mouth and larynx); (2) the first open system to evolve en route to human speech was a manual gestural system that exploited the observation and execution matching system described earlier; and (3) that this paved the way for the evolution of the open vocalization system we know as speech.

As far as the first point is concerned, both F5 and Broca's areas have the neural structures for controlling oro-laryngeal, oro-facial and brachio-manual movements. Furthermore, they are both endowed with mechanisms that link action perception and action production. This is true for brachio-manual gestures (as discussed in this review) as well as, in the case of human Broca's area, for linguistic tasks, including those not requiring speech production ${ }^{39-41}$. It is likely that the human capacity to communicate beyond that of other primates depended on the progressive evolution of the mirror system in its globality. Congruent with this view is the observation by Donald ${ }^{42}$ that mimetic capacity, a natural extension of action recognition, is central to human culture (such as dances, games and tribal rituals), and that the evolution of this capacity was a necessary precursor to the evolution of language.

Even so, it is interesting to speculate on the sequence of events that led from gestural communication to speech. The gestures of primates that were most likely to be first used for person-to-person communication (as distinct from signals that are directed 
to 'everybody', rather than to a specific receiver) are the oro-facial ones. In favor of this view are the following facts: oro-facial movements are used for communication by monkeys, apes and humans, and neither monkeys nor humans use manuo-brachial gestures as their main natural way to communicate. Exceptions are deaf people who naturally use sign language $^{43}$, some Indian populations in North America and some Aboriginal Australian tribes ${ }^{44}$. The openclose alternation of the mandible that is typical of oro-facial communication of monkeys ('lipsmacks', 'tonguesmacks ${ }^{45}$ ) appears to persist in humans where it forms the syllabic 'frame' in speech production ${ }^{46,47}$.

Was speech, therefore, a direct evolution of oro-facial gestures, after which followed an expansion stage during which an open vocalization system appeared? A first reason for doubting this scenario is that by using oro-facial communication, the exchange of communication is essentially limited to two actors. The possibility of introducing a third element in this one-toone communication is very limited. By contrast, if manual gestures are associated with oro-facial communication, the sender's possibilities dramatically increase. The sender might indicate to the receiver the position of a third person or of an object, or even give a certain description of their characteristics. A second reason lies in the fact that the combinatorial properties for the openness of speech are virtually absent in the basic primate form of oro-facial communication. By contrast, they are inherent to the brachio-manual system, both when it is used for transitive actions (actions directed towards objects) and when it is employed for intransitive gestures (as in the case of American Sign Language).

These considerations suggest that, at a certain stage, a brachio-manual communication system evolved complementing the oro-facial one. This development greatly modified the importance of vocalization and its control. Whereas during the closed oro-facial stage, sounds could add very little to the gestural message (for example, oro-facial gesture 'be scared'; oro-facial gesture plus vocalization 'be more scared'), their association with gestures allowed them to assume the more open, referential character that brachiomanual gestures had already achieved ${ }^{48,49}$. An object or event described gesturally (such as, large object - large gesture of the arms, and small object - tiny opening of the fingers) could now be accompanied by vocalization. If identical sounds were constantly used to indicate identical elements (such as, large object - large opening of the mouth, vowel ' $a$ ', and small object tiny opening of the mouth, vowel ' $i$ '), a primitive vocabulary of meaningful sounds could start to develop ${ }^{50,51}$

An important consequence of this new functional use of vocalization was the necessity of its skillful control. In the oro-facial communication system, the addition of a sound had only an emotional valence that simply reinforced the meaning conveyed by the facial expression; its precise execution had a relative importance. Therefore, vocalization could remain under the control of the old system located in the brain medial areas. The situation changed radically when sounds acquired a descriptive value and thus had to remain the same in identical situations and, in addition, had to be imitated when emitted by other individuals. These new requirements could not be ful- filled by the ancient emotional vocalization centers. This new situation was most likely to be the cause of the emergence of human Broca's area from an F5-like precursor that already had mirror properties, a control of oro-laryngeal movements and, in addition, a tight link with the adjacent primary motor cortex. The evolutionary pressure for more complex (combinatorial) sound emission, and the anatomical possibility for it, were thus the elements that moved language from its manuo-brachial origins to sound emission. Manual gestures progressively lost their importance, whereas, by contrast, vocalization acquired autonomy, until the relation between gestural and vocal communication inverted and gesture became purely an accessory factor to sound communication. At this point speech took off.

\section{A historical coda}

The debate on the origin of language has a long history. Clearly we side with those authors who see a common origin for human speech and some forms of communications in primates, with gestural communication playing an important role in human language genesis ${ }^{52-56}$. Chomsky has long argued that language is determined by innate, biologically determined abilities in conjunction with exposure to the language in the environment ${ }^{57}$; to learn a grammar the child must simply use a few fragments of a particular language to 'set' parameters in the Universal Grammar, a genetically determined, biological endowment relevant to language (for a critique of this view see Ref. 58).

Chomsky also downplays the role of natural selection in language evolution. Pinker and Bloom ${ }^{59}$ offer convincing arguments against this view, but their approach to language evolution differs from ours on one major point. They see what has evolved for language as Universal Grammar. Our suggestion, by contrast, is that natural selection yielded a set of generic structures for matching action observation and execution. These structures, coupled with appropriate learning mechanisms, proved great enough to support cultural evolution of human languages in all their richness. We hold that human language (as well as some dyadic forms of primate communication) evolved from a basic mechanism that was not originally related to communication: the capacity to recognize actions.

Imprints in fossil cranial cavities indicate that 'speech areas' were already present in early hominids such as Homo habilis (Ref. 60), but there is debate over whether or not such areas were already present ${ }^{61}$ or not $^{62}$ in australopithecines. A plausible hypothesis is that the transition from the australopithecines to the first forms of 'Homo' coincided with the transition from a mirror system, enlarged, but used only for action recognition, to a human-like mirror system used for intentional communication. Our view on the subsequent scenario is close to that of Corballis ${ }^{53}$. The 'proto-speech' areas of early hominids mediated orofacial and brachio-manual communication, but not speech. The long period from the appearance of these areas to the appearance of speech ${ }^{63,64}$ coincided with an increased capacity to communicate with gesture and the progressive association of gesture with vocalization.

In conclusion, the discovery of the mirror system suggests a strong link between speech and action representation. 'One sees a distinctly linguistic way of 
doing things down among the nuts and bolts of action and perception, for it is there, not in the remote recesses of cognitive machinery, that the specifically linguistic constituents make their first appearance ${ }^{11}$.

\section{Selected references}

1 Liberman, A.M. (1993) Haskins Laboratories Status Report on Speech Research 113, 1-32

2 Kurata, K. and Tanji, J. (1986) J. Neurosci. 6, 403-411

3 Rizzolatti, G. et al. (1988) Exp. Brain Res. 71, 491-507

4 Hepp-Reymond, M-C. et al. (1994) Can. J. Physiol. Pharmacol. 72, 571-579

5 Jeannerod, M. et al. (1995) Trends Neurosci. 18, 314-320

6 Di Pellegrino, G. et al. (1992) Exp. Brain Res. 91, 176-180

7 Rizzolatti, G. et al. (1996) Cogn. Brain Res. 3, 131-141

8 Gallese, V. et al. (1996) Brain 119, 593-609

9 Jeannerod, M. (1994) Behav. Brain Sci. 17, 187-245

10 Liberman, A.M. and Mattingly, I.G. (1985) Cognition 21, 1-36

11 Liberman, A.M. and Mattingly, I.G. (1989) Science 243, 489-494

12 Passingham, R. (1981) Philos. Trans. R. Soc. London Ser. B 292, 167-175

13 Campbell, A.W. (1905) Histological Studies on the Localisation of Cerebral Function, Cambridge University Press

14 Von Bonin, G. and Bailey, P. (1947) The Neocortex of Macaca Mulatta, University of Illinois Press

15 Passingham, R. (1993) The Frontal Lobes and Voluntary Action, Oxford University Press

16 Galaburda, A.M. and Pandya, D.N. (1982) in Primate Brain Evolution (Armstrong, E. and Falk, D., eds), pp. 203-216, Plenum Press

17 Petrides, M. and Pandya, D.N. (1994) in Handbook of Neuropsychology (Vol. IX) (Boller, F. and Grafman, J., eds), pp. 17-58, Elsevier

18 Rizzolatti, G. et al. (1981) Behav. Brain Res. 2, 147-163

19 Hast, M.H. et al. (1974) Brain Res. 73, 229-240

20 Gentilucci, M. et al. (1988) Exp. Brain Res. 71, 475-490

21 Preuss, T.M. (1995) in The Cognitive Neurosciences (Gazzaniga, M.S., ed.), pp. 1227-1241, MIT Press

22 Bonda, E. et al. (1994) Soc. Neurosci. Abstr. 20, 353

23 Schlaug, G., Knorr, U. and Seitz, R.J. (1994) Exp. Brain Res. 98, 523-534

24 Decety, J. et al. (1994) Nature 371, 600-602

25 Grafton, S.T. et al. (1996) Exp. Brain Res. 112, 103-111

26 Parsons, L.M. et al. (1995) Nature 375, 54-58

27 Chollet, F. et al. (1991) Ann. Neurol. 29, 63-71

28 Fadiga, L. et al. (1995) J. Neurophysiol. 73, 2608-2611

29 Rizzolatti, G. et al. (1996) Exp. Brain Res. 111, 246-252

30 Perrett, D.I. et al. (1989) J. Exp. Biol. 146, 87-113

31 Perrett, D.I. et al. (1990) in Vision and Action: The Control of Grasping (Goodale, M.A., ed.), pp. 163-180, Ablex

32 Leinonen, L. and Nyman, G. (1979) Exp. Brain Res. 34, 321-333

33 Jurgens, U. (1995) in Current Topics in Primate Vocal Communication (Zimmerman, E., Newman, J.D. and Jurgens, U., eds), pp. 199-206, Plenum Press

34 MacLean, P.D. (1993) in Neurobiology of Cingulate Cortex and Limbic Thalamus: A Comprehensive Handbook (Vogt, B.A. and Gabriel, M., eds), pp. 1-15, Birkhäuse

35 Arbib, M.A. and Rizzolatti, G. (1997) Commun. Cogn. 29, 393-424

36 Fillmore, C.J. (1966) in Universals in Linguistic Theory (Bach, E. and Harms, R.T., eds.), pp. 1-88, Rinehart and Winston

37 van Riemsdijk, H. and Williams, E. (1986) Introduction to the Theory of Grammar, MIT Press

38 Iberall, T. et al. (1986) Exp. Brain Res. 15, 158-173

39 Demonet, J-F. et al. (1992) Brain 115, 1753-1768

40 Zatorre, R.J. et al. (1992) Science 256, 846-849

41 Demonet, J-F., Wise, R. and Frackowiak, R.S.J. (1993) Hum. Brain Mapp. 1, 39-47

42 Donald, M. (1991) Origins of the Modern Mind: Three Stages in the Evolution of Culture and Cognition, Harvard University Press

43 Klima, E.S. and Bellugi, U. (1979) The Signs of the Language, Harvard University Press

44 Kendon, A. (1988) Sign Languages of Aboriginal Australians, Cambridge University Press

45 Redican, W.K. (1975) in Primate Behavior: Developments in Field and Laboratory Research (Vol. 4) (Rosenblum, L.A., ed.), pp. 104-194, Academic Press

46 MacNeilage, P.F. (1997) in Approaches to the Evolution of Language (Hurford, J.R., Knight, C. and Studdert-Kennedy, M.G., eds), pp. 234-252, Cambridge University Press

47 MacNeilage, P.F. Behav. Brain Sci. (in press)

48 Paget, R.A.S. (1963) Human Speech: Some Observations, Experiments and Conclusions as to the Nature, Origin, Purpose and Possible Improvement of Human Speech, Routledge and Kegan Paul 49 Johanesson, A. (1950) Nature 166, 60-61

50 Holland, M. and Wertheimer, M. (1964) Percept. Motor Skill 19, 111-117

51 Weiss, J.H. (1964) Psychol. Bull. 61, 454-458

52 Hewes, G.W. (1973) Curr. Anthropol. 14, 5-24

53 Corballis, M.C. (1992) Cognition 44, 197-226

54 Corballis, M.C. (1991) The Lopsided Ape: Evolution of the Generative Mind, Oxford University Press

55 Kimura, D. (1993) Neuromotor Mechanisms in Human Communication, Oxford University Press

56 Armstrong, D.F., Stokoe, W.C. and Wilcox, S.E. (1995) Gesture and the Nature of Language, Cambridge University Press

57 Chomsky, N. (1986) Knowledge of Language: Its Nature, Origin and Use, Praeger

58 Arbib, M.A. and Hill J.C. (1988) in Explaining Language Universals (Hawkins, J.A., ed.), pp. 56-72, Blackwell

59 Pinker, S. and Bloom, P. (1990) Behav. Brain Sci. 12, 707-784

60 Tobias, P.V. (1987) J. Hum. Evol. 16, 741-761

61 Holloway, R.L. (1981) Am. J. Phys. Anthropol. 56, 43-58

62 Falk, D. (1983) Science 222, 1072-1074

63 Lieberman, P., Crelin, E.S. and Klatt, D.H. (1972) Am. Anthropol. 74, 287-307

64 Lieberman, P. (1984) The Biology and Evolution of Language, Harvard University Press

\section{In the other Trends journals}

Ataxia, arrythmia and ion-channel defects, by Johannah L. Doyle and Lisa Stubbs Trends in Genetics I4, 92-98

Genetic blindness: current concepts in the pathogenesis of human outer retinal dystrophies, by Kevin Gregory-Evans and Shomi S. Battacharya Trends in Genetics 14, 103-108

Inhibition of TNF-induced apoptosis by NF- $\mathrm{B}$,

by Daniel J. Van Antwerp, Seamus J. Martin, Inder Verma and Douglas R. Green Trends in Cell Biology 8, I07-III

New aspects of G-protein-coupled receptor signalling and regulation, by Graeme Milligan

Trends in Endocrinology and Metabolism 9, 13-19

Neural basis of motor control and its cognitive implications, by Emilio Bizzi and Ferdinando A. Mussa-Ivaldi Trends in Cognitive Sciences 2, 97-102

Magnetic stimulation studies of visual cognition, by Vincent Walsh and Alan Cowey Trends in Cognitive Sciences, 2, 103-110

Endocannabinoids: a new class of vasoactive substances, by M.D. Randall and D.A. Kendall Trends in Pharmacological Sciences 19, 55-58 